\title{
Temores y desafíos actuales sobre la Radiología Oral y Maxilofacial
}

\author{
Current fears and challenges over Oral and Maxillofacial Radiology.
}

\section{Sr. Editor:}

Con mucho interés he leído la carta al editor de los colegas Daniel Atau Mollo y Vilma Elizabeth Ruiz García de Chacón, publicada en el volumen 28, número 3 de la Revista Estomatológica Herediana, en relación a la relevancia del comportamiento de los pacientes respecto a las radiaciones ionizantes (1).

El temor que la radiación ionizante produce en los pacientes complejiza la atención en los servicios de imagenología. Existe un creciente temor por parte de la población general sobre los efectos que las radiografías dentales y las tomografías de haz cónico tienen sobre el cáncer de tiroides. El aumento de su incidencia ha dado pie a artículos, notas y blogs de fácil acceso, sin la debida rigurosidad científica, con el fin de "informar" respecto a este tipo de exámenes, generando un temor que termina por comprometer el diagnóstico oportuno de patologías dentales y maxilofaciales. El recelo que produce la oración "un estudio demostró que" en el lector no científico, resulta particularmente difícil de subsanar. Los conceptos de dosis efectiva, radiosensibilidad, efectos determinísticos y no determinísticos, que son parte esencial de la discusión sobre el riesgo radiológico, son tópicos omitidos en los "reportajes en salud", disminuyendo la percepción de seguridad por parte de los pacientes. Tampoco se hace hincapié en los avances tecnológicos y la sistemática reducción de los tiempos de expo- sición, entre otros, sesgando la información al lector sobre el examen radiológico (2).

La discusión científica sobre la relación del cáncer de tiroides y las radiografías dentales en la también merece prudencia. En su volumen 208, número 12, el Bristish Dental Journal cita un trabajo realizado por Anjum Memon, Sara Godward, Dillwyn Williams, Iqbal Siddique y Khalid Al- Saleh, en un trabajo colaborativo entre Reino Unido y Kuwait, el cual consistió en un estudio de caso y control sobre el aumento en la incidencia del cáncer de tiroides, encontrado un aumento del riesgo entre este y los examenes imagenológicos dentales (3). La American Cáncer Society describe como factor de riesgo del cáncer de tiroides las altas radiaciones ionizantes a temprana edad, como es el caso de las radioterapias, y estimó un aumento de este cáncer en 53.999 casos nuevos para él, en ese entonces, año 2018 (4); este dato en poder de fuentes de información ajenas a la odontología supone una potencial barrera para, por ejemplo, una correcta planificación de tratamientos pediátricos, dada la mayor aprensión por parte de madre y padre (5). Toda evidencia debe ser tomada bajo una mirada analítica. Serán necesarias revisiones sistemáticas con metodologías de búsqueda y criterios de inclusión depurados, para verificar si la relación entre radiología oral y maxilofacial y el cáncer de tiroides es consistente. Por lo tanto, a la luz de una evidencia que puede abrumar al paciente que busca información, educar a 
la comunidad sobre las dosis de radiación en relación al riesgo-beneficio es una tarea pendiente, tal como se expone en la carta al editor referenciada (1).

\section{José Pablo Tisi ${ }^{1, a}$}

\section{Declaración de Financiamiento y Conflicto de interés:}

El presente trabajo fue realizado sin ningún tipo de financiamiento. No hay conflicto de interés.

\section{Correspondencia:}

José Pablo Tisi.

Correo Electrónico: j.pablo.tisi@gmail.com

\section{REFERENCIAS BIBLIOGRÁFICAS}

1. Atau D, Ruiz V. Conocimiento y percepción de riesgo de los usuarios sobre los exámenes imagenologicos: ¿influye en la decisión para el procedimiento? Rev Estomatol Herediana. 2018; 28(3): 287-88. DOI: https://doi.org/10.20453/reh.v28i4.3434

2. Ubeda C, Nocetti D, Aragón M. Seguridad y protección radiológica en procedimientos imagenológicos dentales. Int. J. Odontostomat. 2018; 12(3):246-251.

3. Memon A, Godward S, Williams D, Siddique I, AlSaleh K. Dental X-rays and the risk of thyroid cancer: A case-control study. Acta Oncol. 2010; 49: 447-543.

4. American Cancer Society. Cancer Facts \& Figures. Atlanta: American Cancer Society; 2018. p. 24.

5. Coşkun I, Kaya B. Cone Beam Computed Tomography in orthodontics. Turk J Ortod. 2018; 31: 55-61.

Recibido: 16-08-2019 